\title{
The Calculation of New Prime Number Theorem Formula
}

\section{YinYue Sha*}

Dongling Engineering Center, Ningbo Institute of Technology, Zhejiang University, PR China

\section{Short Commentary}

Let $\mathrm{Pi}(\mathrm{N})$ be the prime - counting function that gives the number of primes less than or equal to $\mathrm{N}$, for any real number $\mathrm{N}$, then new prime number theorem can be expressed by the formula as follows: $-1\}$,

$\mathrm{Pi}(\mathrm{N})=\operatorname{INT}\{\mathrm{N} \times(1-1 / \mathrm{P} 1) \times(1-1 / \mathrm{P} 2) \times \ldots \times(1-1 / \mathrm{Pm})+\mathrm{m}$

where the INT $\{\ldots\}$ expresses the taking integer operation of formula spread out type in $\{\ldots\}, \mathrm{P} 1, \mathrm{P} 2, \ldots, \mathrm{Pm}$ are all prime numbers less than or equal to $\sqrt{N}$.

According to the above formula we can obtain the formula as follows:

$\operatorname{Pi}(\mathrm{N}) \approx \operatorname{Psha}(\mathrm{N}) \equiv \operatorname{Li}(\mathrm{N}) \times(1-(1+1 /(\operatorname{Ln}(\mathrm{N})-5)) / \sqrt{N})$

$\geq \operatorname{Sha}(\mathrm{N}) \equiv 2 /(1+\sqrt{ }(1-4 / \operatorname{Ln}(\mathrm{N}))) \times \mathrm{N} / \operatorname{Ln}(\mathrm{N}) \geq \mathrm{N} /(\operatorname{Ln}(\mathrm{N})-1)$,

where $\operatorname{Li}(\mathrm{N})$ is the logarithmic integral function, the $\operatorname{Ln}(\mathrm{N})$ denotes the natural logarithm of $\mathrm{N}$.

One: The Peculiarity of New Prime Number Theorem Formula

Let P1, P2, .., Pn are all prime numbers less than or equal to Pn, then exists the formula as follows:

$\mathrm{INT}\{\mathrm{Ni} \times(1-1 / \mathrm{P} 1) \times(1-1 / \mathrm{P} 2) \times(1-1 / \mathrm{P} 3) \times \ldots \ldots \times(1-1 /$ $\mathrm{Pn})-1\}=0,1 \leq \mathrm{Ni} \leq \mathrm{Pn}$

For example:

INT $\{1-1\}=\operatorname{INT}\{1-1\}=\{1-1\}=0$

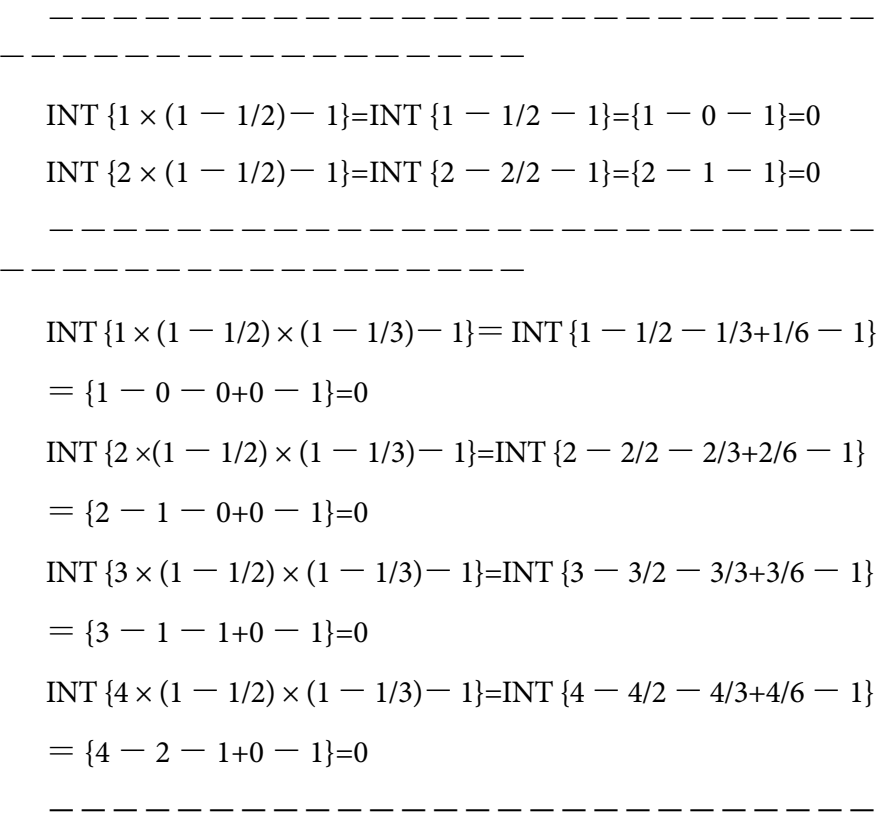

$\operatorname{INT}\{1 \times(1-1 / 2) \times(1-1 / 3) \times(1-1 / 5)-1\}$

$=\operatorname{INT}\{1-1 / 2-1 / 3-1 / 5+1 / 6+1 / 10+1 / 15-1 / 30-1\}$

$$
=\{1-0-0-0+0+0+0-0-1\}=0
$$

INT $\{2 \times(1-1 / 2) \times(1-1 / 3) \times(1-1 / 5)-1\}$

$=\operatorname{INT}\{2-2 / 2-2 / 3-2 / 5+2 / 6+2 / 10+2 / 15-2 / 30-1\}$

$=\{2-1-0-0+0+0+0-0-1\}=0$

INT $\{3 \times(1-1 / 2) \times(1-1 / 3) \times(1-1 / 5)-1\}$

$=\operatorname{INT}\{3-3 / 2-3 / 3-3 / 5+3 / 6+3 / 10+3 / 15-3 / 30-1\}$

$=\{3-1-1-0+0+0+0-0-1\}=0$

INT $\{4 \times(1-1 / 2) \times(1-1 / 3) \times(1-1 / 5)-1\}$

$=\operatorname{INT}\{4-4 / 2-4 / 3-4 / 5+4 / 6+4 / 10+4 / 15-4 / 30-1\}$

$=\{4-2-1-0+0+0+0-0-1\}=0$

INT $\{5 \times(1-1 / 2) \times(1-1 / 3) \times(1-1 / 5)-1\}$

$=\operatorname{INT}\{5-5 / 2-5 / 3-5 / 5+5 / 6+5 / 10+5 / 15-5 / 30-1\}$

$=\{5-2-1-1+0+0+0-0-1\}=0$

INT $\{6 \times(1-1 / 2) \times(1-1 / 3) \times(1-1 / 5)-1\}$

$=\operatorname{INT}\{6-6 / 2-6 / 3-6 / 5+6 / 6+6 / 10+6 / 15-6 / 30-1\}$

$=\{6-3-2-1+1+0+0-0-1\}=0$

Two: The Calculation of New Prime Number Theorem Formula

$\mathrm{Pi}(\mathrm{N})=\operatorname{INT}\{\mathrm{N} \times(1-1 / \mathrm{P} 1) \times(1-1 / \mathrm{P} 2) \times(1-1 / \mathrm{P} 3) \times \ldots \ldots \times$ $(1-1 / \mathrm{Pm})+\mathrm{m}-1\}$

For example:

$$
\begin{aligned}
& \operatorname{Pi}(1)=\operatorname{INT}\{1-1\}=\{1-1\}=0 \\
& \operatorname{Pi}(2)=\operatorname{INT}\{2-1\}=\{2-1\}=1 \\
& \operatorname{Pi}(3)=\operatorname{INT}\{3--1\}=\{3-1\}=2
\end{aligned}
$$

$$
\begin{aligned}
& \operatorname{Pi}(2)=\operatorname{INT}\{2 \times(1-1 / 2)+1-1\} \\
& =\operatorname{INT}\{2-2 / 2+1-1\}=\{2-1+1-1\}=1 \\
& \operatorname{Pi}(3)=\operatorname{INT}\{3 \times(1-1 / 2)+1-1\} \\
& =\operatorname{INT}\{3-3 / 2+1-1\}=\{3-1+1-1\}=2
\end{aligned}
$$

*Corresponding author: YinYue Sha, Dongling Engineering Center, Ningbo Institute of Technology, Zhejiang University, PR China, Tel: 5718517 2244; E-mail: shayinyue@qq.com

Received September 27, 2017; Accepted October 17, 2017; Published October 25, 2017

Citation: Sha YY (2017) The Calculation of New Prime Number Theorem Formula. J Generalized Lie Theory Appl 11: 283. doi: 10.4172/1736-4337.1000283

Copyright: (c) 2017 Sha YY. This is an open-access article distributed under the terms of the Creative Commons Attribution License, which permits unrestricted use, distribution, and reproduction in any medium, provided the original author and source are credited. 
Citation: Sha YY (2017) The Calculation of New Prime Number Theorem Formula. J Generalized Lie Theory Appl 11: 283. doi: 10.4172/17364337.1000283

Page 2 of 4

$$
\begin{aligned}
& \operatorname{Pi}(4)=\operatorname{INT}\{4 \times(1-1 / 2)+1-1\} \\
& =\operatorname{INT}\{4-4 / 2+1-1\}=\{4-2+1-1\}=2 \\
& \operatorname{Pi}(5)=\operatorname{INT}\{5 \times(1-1 / 2)+1-1\} \\
& =\operatorname{INT}\{5-5 / 2+1-1\}=\{5-2+1-1\}=3 \\
& \operatorname{Pi}(6)=\operatorname{INT}\{6 \times(1-1 / 2)+1-1\} \\
& =\operatorname{INT}\{6-6 / 2+1-1\}=\{6-3+1-1\}=3 \\
& \operatorname{Pi}(7)=\operatorname{INT}\{7 \times(1-1 / 2)+1-1\} \\
& =\operatorname{INT}\{7-7 / 2+1-1\}=\{7-3+1-1\}=4 \\
& \operatorname{Pi}(8)=\operatorname{INT}\{8 \times(1-1 / 2)+1-1\} \\
& =\operatorname{INT}\{8-8 / 2+1-1\}=\{8-4+1--1\}=4
\end{aligned}
$$$$
---------------------
$$$$
\operatorname{Pi}(3)=\operatorname{INT}\{3 \times(1-1 / 2) \times(1-1 / 3)+2-1\}
$$$$
=\operatorname{INT}\{3-3 / 2-3 / 3+3 / 6+2-1\}=\{3-1-1+0+2-1\}=2
$$$$
\operatorname{Pi}(4)=\operatorname{INT}\{4 \times(1-1 / 2) \times(1-1 / 3)+2-1\}
$$$$
=\operatorname{INT}\{4-4 / 2-4 / 3+4 / 6+2-1\}=\{4-2-1+0+2-1\}=2
$$$$
\operatorname{Pi}(5)=\operatorname{INT}\{5 \times(1-1 / 2) \times(1-1 / 3)+2-1\}
$$$$
=\operatorname{INT}\{5-5 / 2-5 / 3+5 / 6+2-1\}=\{5-2-1+0+2-1\}=3
$$$$
\operatorname{Pi}(6)=\operatorname{INT}\{6 \times(1-1 / 2) \times(1-1 / 3)+2-1\}
$$$$
=\operatorname{INT}\{6-6 / 2-6 / 3+6 / 6+2-1\}=\{6-3-2+1+2-1\}=3
$$$$
\operatorname{Pi}(7)=\operatorname{INT}\{7 \times(1-1 / 2) \times(1-1 / 3)+2-1\}
$$$$
=\operatorname{INT}\{7-7 / 2-7 / 3+7 / 6+2-1\}=\{7-3-2+1+2-1\}=4
$$$$
\operatorname{Pi}(8)=\operatorname{INT}\{8 \times(1-1 / 2) \times(1-1 / 3)+2-1\}
$$$$
=\operatorname{INT}\{8-8 / 2-8 / 3+8 / 6+2-1\}=\{8-4-2+1+2-1\}=4
$$$$
\operatorname{Pi}(9)=\operatorname{INT}\{9 \times(1-1 / 2) \times(1-1 / 3)+2-1\}
$$$$
=\operatorname{INT}\{9-9 / 2-9 / 3+9 / 6+2-1\}=\{9-4-3+1+2-1\}=4
$$$$
\operatorname{Pi}(10)=\operatorname{INT}\{10 \times(1-1 / 2) \times(1-1 / 3)+2-1\}
$$$$
=\operatorname{INT}\{10-10 / 2-10 / 3+10 / 6+2-1\}=\{10-5-3+1+2-1\}=4
$$$$
\operatorname{Pi}(11)=\operatorname{INT}\{11 \times(1-1 / 2) \times(1-1 / 3)+2-1\}
$$$$
=\operatorname{INT}\{11-11 / 2-11 / 3+11 / 6+2-1\}=\{11-5-3+1+2-1\}=5
$$$$
\operatorname{Pi}(12)=\operatorname{INT}\{12 \times(1-1 / 2) \times(1-1 / 3)+2-1\}
$$$$
=\operatorname{INT}\{12-12 / 2-12 / 3+12 / 6+2-1\}=\{12-6-4+2+2-1\}=5
$$$$
\operatorname{Pi}(13)=\operatorname{INT}\{13 \times(1-1 / 2) \times(1-1 / 3)+2-1\}
$$$$
=\operatorname{INT}\{13-13 / 2-13 / 3+13 / 6+2-1\}=\{13-6-4+2+2-1\}=6
$$$$
\operatorname{Pi}(14)=\operatorname{INT}\{14 \times(1-1 / 2) \times(1-1 / 3)+2-1\}
$$$$
=\operatorname{INT}\{14-14 / 2-14 / 3+14 / 6+2-1\}=\{14-7-4+2+2-1\}=6
$$$$
\operatorname{Pi}(15)=\operatorname{INT}\{15 \times(1-1 / 2) \times(1-1 / 3)+2-1\}
$$$$
=\operatorname{INT}\{15-15 / 2-15 / 3+15 / 6+2-1\}=\{15-7-5+2+2-1\}=6
$$$$
\operatorname{Pi}(16)=\operatorname{INT}\{16 \times(1-1 / 2) \times(1-1 / 3)+2-1\}
$$$$
=\operatorname{INT}\{16-16 / 2-16 / 3+16 / 6+2-1\}=\{16-8-5+2+2-1\}=6
$$

$\operatorname{Pi}(17)=\operatorname{INT}\{17 \times(1-1 / 2) \times(1-1 / 3)+2-1\}$

$=\operatorname{INT}\{17-17 / 2-17 / 3+17 / 6+2-1\}=\{17-8-5+2+2-1\}=7$ $\operatorname{Pi}(18)=\operatorname{INT}\{18 \times(1-1 / 2) \times(1-1 / 3)+2-1\}$

$=\operatorname{INT}\{18-18 / 2-18 / 3+18 / 6+2-1\}=\{18-9-6+3+2-1\}=7$ $\operatorname{Pi}(19)=\operatorname{INT}\{19 \times(1-1 / 2) \times(1-1 / 3)+2-1\}$

$=\operatorname{INT}\{19-19 / 2-19 / 3+19 / 6+2-1\}=\{19-9-6+3+2-1\}=8$ $\operatorname{Pi}(20)=\operatorname{INT}\{20 \times(1--1 / 2) \times(1-1 / 3)+2-1\}$

$=\operatorname{INT}\{20-20 / 2-20 / 3+20 / 6+2-1\}=\{20-10-6+3+2-1\}=8$ $\operatorname{Pi}(21)=\operatorname{INT}\{21 \times(1-1 / 2) \times(1-1 / 3)+2-1\}$

$=\operatorname{INT}\{21-21 / 2-21 / 3+21 / 6+2-1\}=\{21-10-7+3+2-1\}=8$ $\operatorname{Pi}(22)=\operatorname{INT}\{22 \times(1-1 / 2) \times(1-1 / 3)+2-1\}$

$=\operatorname{INT}\{22-22 / 2-22 / 3+22 / 6+2-1\}=\{22-11-7+3+2-1\}=8$ $\operatorname{Pi}(23)=\operatorname{INT}\{23 \times(1-1 / 2) \times(1-1 / 3)+2-1\}$

$=\operatorname{INT}\{23-23 / 2-23 / 3+23 / 6+2-1\}=\{23-11-7+3+2-1\}=9$ $\operatorname{Pi}(24)=\operatorname{INT}\{24 \times(1-1 / 2) \times(1-1 / 3)+2-1\}$

$=\operatorname{INT}\{24-24 / 2-24 / 3+24 / 6+2-1\}=\{24-12-8+4+2-1\}=9$

$\operatorname{Pi}(5)=\operatorname{INT}\{5 \times(1-1 / 2) \times(1-1 / 3) \times(1-1 / 5)+3-1\}$

$=\operatorname{INT}\{5-5 / 2-5 / 3-5 / 5+5 / 6+5 / 10+5 / 15-5 / 30+3-1\}$

$=\{5-2-2-1+1+0+0-0+3-1\}=3$

$\operatorname{Pi}(6)=\operatorname{INT}\{6 \times(1-1 / 2) \times(1-1 / 3) \times(1-1 / 5)+3-1\}$

$=\operatorname{INT}\{6-6 / 2-6 / 3-6 / 5+6 / 6+6 / 10+6 / 15-6 / 30+3-1\}$

$=\{6-3-2-1+1+0+0-0+3-1\}=3$

$\operatorname{Pi}(7)=\operatorname{INT}\{7 \times(1-1 / 2) \times(1-1 / 3) \times(1-1 / 5)+3-1\}$

$=\operatorname{INT}\{7-7 / 2-7 / 3-7 / 5+7 / 6+7 / 10+7 / 15-7 / 30+3-1\}$

$=\{7-3-2-1+1+0+0-0+3-1\}=4$

$\operatorname{Pi}(8)=\operatorname{INT}\{8 \times(1-1 / 2) \times(1-1 / 3) \times(1-1 / 5)+3-1\}$

$=\operatorname{INT}\{8-8 / 2-8 / 3-8 / 5+8 / 6+8 / 10+8 / 15-8 / 30+3-1\}$

$=\{8-4-2-1+1+0+0-0+3-1\}=4$

$\operatorname{Pi}(9)=\operatorname{INT}\{9 \times(1-1 / 2) \times(1-1 / 3) \times(1-1 / 5)+3-1\}$

$=\operatorname{INT}\{9-9 / 2-9 / 3-9 / 5+9 / 6+9 / 10+9 / 15-9 / 30+3-1\}$

$=\{9-4-3-1+1+0+0-0+3-1\}=4$

$\operatorname{Pi}(10)=\operatorname{INT}\{10 \times(1-1 / 2) \times(1-1 / 3) \times(1-1 / 5)+3-1\}$

$=\operatorname{INT}\{10-10 / 2-10 / 3-10 / 5+10 / 6+10 / 10+10 / 15-10 / 30+3-1\}$

$=\{10-5-3-2+1+1+0-0+3-1\}=4$

$\operatorname{Pi}(11)=\operatorname{INT}\{11 \times(1-1 / 2) \times(1-1 / 3) \times(1-1 / 5)+3-1\}$

$=\operatorname{INT}\{11-11 / 2-11 / 3-11 / 5+11 / 6+11 / 10+11 / 1511 / 30+3-1\}$

$=\{11-5-3-2+1+1+0-0+3-1\}=5$

$\operatorname{Pi}(12)=\operatorname{INT}\{12 \times(1-1 / 2) \times(1-1 / 3) \times(1-1 / 5)+3-1\}$ 
Citation: Sha YY (2017) The Calculation of New Prime Number Theorem Formula. J Generalized Lie Theory Appl 11: 283. doi: 10.4172/17364337.1000283

Page 3 of 4

$$
\begin{aligned}
& =\operatorname{INT}\{12-12 / 2-12 / 3-12 / 5+12 / 6+12 / 10+12 / 15-12 / 30+3-1\} \\
& =\{12-6-4-2+2+1+0-0+3-1\}=5 \\
& \operatorname{Pi}(13)=\operatorname{INT}\{13 \times(1-1 / 2) \times(1-1 / 3) \times(1-1 / 5)+3-1\} \\
& =\operatorname{INT}\{13-13 / 2-13 / 3-13 / 5+13 / 6+13 / 10+13 / 15-13 / 30+3-1\} \\
& =\{13-6-4-2+2+1+0-0+3-1\}=6 \\
& \operatorname{Pi}(14)==\operatorname{INT}\{14 \times(1-1 / 2) \times(1-1 / 3) \times(1-1 / 5)+3-1\} \\
& =\operatorname{INT}\{14-14 / 2-14 / 3-14 / 5+14 / 6+14 / 10+14 / 15-14 / 30+3-1\} \\
& =\{14-7-4-2+2+1+0-0+3-1\}=6 \\
& \operatorname{Pi}(15)=\operatorname{INT}\{15 \times(1-1 / 2) \times(1-1 / 3) \times(1-1 / 5)+3-1\} \\
& =\operatorname{INT}\{15-15 / 2-15 / 3-15 / 5+15 / 6+15 / 10+15 / 15-15 / 30+3-1\} \\
& =\{15-7-5-3+2+1+1-0+3-1\}=6 \\
& \operatorname{Pi}(16)=\operatorname{INT}\{16 \times(1-1 / 2) \times(1-1 / 3) \times(1-1 / 5)+3-1\} \\
& =\operatorname{INT}\{16-16 / 2-16 / 3-16 / 5+16 / 6+16 / 10+16 / 15-16 / 30+3-1\} \\
& =\{16-8-5-3+2+1+1-0+3-1\}=6 \\
& \operatorname{Pi}(17)=\operatorname{INT}\{17 \times(1-1 / 2) \times(1-1 / 3) \times(1-1 / 5)+3-1\} \\
& =\operatorname{INT}\{17-17 / 2-17 / 3-17 / 5+17 / 6+17 / 10+17 / 15-17 / 30+3-1\} \\
& =\{17-8-5-3+2+1+1-0+3-1\}=7 \\
& \operatorname{Pi}(18)=\operatorname{INT}\{18 \times(1-1 / 2) \times(1-1 / 3) \times(1-1 / 5)+3-1\} \\
& =\operatorname{INT}\{18-18 / 2-18 / 3-18 / 5+18 / 6+18 / 10+18 / 15-18 / 30+3-1\} \\
& =\{18-9-6-3+3+1+1-0+3-1\}=7 \\
& \operatorname{Pi}(19)=\operatorname{INT}\{19 \times(1-1 / 2) \times(1-1 / 3) \times(1-1 / 5)+3-1\} \\
& =\operatorname{INT}\{19-19 / 2-19 / 3-19 / 5+19 / 6+19 / 10+19 / 15-19 / 30+3-1\} \\
& =\{19-9-6-3+3+1+1-0+3-1\}=8 \\
& \operatorname{Pi}(20)=\operatorname{INT}\{20 \times(1-1 / 2) \times(1-1 / 3) \times(1-1 / 5)+3-1\} \\
& =\operatorname{INT}\{20-20 / 2-20 / 3-20 / 5+20 / 6+20 / 10+20 / 15-20 / 30+3-1\} \\
& =\{20-10-6-4+3+2+1-0+3-1\}=8 \\
& \operatorname{Pi}(21)=\operatorname{INT}\{21 \times(1-1 / 2) \times(1-1 / 3) \times(1-1 / 5)+3-1\} \\
& =\operatorname{INT}\{21-21 / 2-21 / 3-21 / 5+21 / 6+21 / 10+21 / 15-21 / 30+3-1\} \\
& =\{21-10-7-4+3+2+1-0+3-1\}=8 \\
& \operatorname{Pi}(22)=\operatorname{INT}\{22 \times(1-1 / 2) \times(1-1 / 3) \times(1-1 / 5)+3-1\} \\
& =\operatorname{INT}\{22-22 / 2-22 / 3-22 / 5+22 / 6+22 / 10+22 / 15-22 / 30+3-1\} \\
& =\{22-11-7-4+3+2+1-0+3-1\}=8 \\
& \operatorname{Pi}(23)=\operatorname{INT}\{23 \times(1-1 / 2) \times(1-1 / 3) \times(1-1 / 5)+3-1\} \\
& =\text { INT }\{23-23 / 2-23 / 3-23 / 5+23 / 6+23 / 10+23 / 15-- \\
& 23 / 30+3-1\} \\
& =\{23-11-7-4+3+2+1-0+3-1\}=9 \\
& \operatorname{Pi}(24)=\operatorname{INT}\{24 \times(1-1 / 2) \times(1-1 / 3) \times(1-1 / 5)+3-1\} \\
& =\operatorname{INT}\{24-24 / 2-24 / 3-24 / 5+24 / 6+24 / 10+24 / 15-24 / 30+3-1\} \\
& =\{24-12-8-4+4+2+1-0+3-1\}=9 \\
& \operatorname{Pi}(25)=\operatorname{INT}\{25 \times(1-1 / 2) \times(1-1 / 3) \times(1-1 / 5)+3-1\}
\end{aligned}
$$

$=\operatorname{INT}\{25-25 / 2-25 / 3-25 / 5+25 / 6+25 / 10+25 / 15-25 / 30+3-1\}$ $=\{25-12-8-5+4+2+1-0+3-1\}=9$

$\operatorname{Pi}(26)=\operatorname{INT}\{26 \times(1-1 / 2) \times(1-1 / 3) \times(1-1 / 5)+3-1\}$

$=\operatorname{INT}\{26-26 / 2-26 / 3-26 / 5+26 / 6+26 / 10+26 / 15-26 / 30+3-1\}$

$=\{26-13-8-5+4+2+1-0+3-1\}=9$

$\operatorname{Pi}(27)=\operatorname{INT}\{27 \times(1-1 / 2) \times(1-1 / 3) \times(1-1 / 5)+3-1\}$

$=\operatorname{INT}\{27-27 / 2-27 / 3-27 / 5+27 / 6+27 / 10+27 / 15-27 / 30+3-1\}$

$=\{27-13-9-5+4+2+1-0+3-1\}=9$

$\operatorname{Pi}(28)=\operatorname{INT}\{28 \times(1-1 / 2) \times(1-1 / 3) \times(1-1 / 5)+3-1\}$

$=\operatorname{INT}\{28-28 / 2-28 / 3-28 / 5+28 / 6+28 / 10+28 / 15-28 / 30+3-1\}$

$=\{28-14-9-5+4+2+1-0+3-1\}=9$

$\operatorname{Pi}(29)=\operatorname{INT}\{29 \times(1-1 / 2) \times(1-1 / 3) \times(1-1 / 5)+3-1\}$

$=\operatorname{INT}\{29-29 / 2-29 / 3-29 / 5+29 / 6+29 / 10+29 / 15-29 / 30+3-1\}$

$=\{29-14-9-5+4+2+1-0+3-1\}=10$

$\operatorname{Pi}(30)=\operatorname{INT}\{30 \times(1-1 / 2) \times(1-1 / 3) \times(1-1 / 5)+3-1\}$

$=\operatorname{INT}\{30-30 / 2-30 / 3-30 / 5+30 / 6+30 / 10+30 / 15-30 / 30+3-1\}$

$=\{30-15-10-6+5+3+2-1+3-1\}=10$

$\operatorname{Pi}(31)=\operatorname{INT}\{31 \times(1-1 / 2) \times(1-1 / 3) \times(1-1 / 5)+3-1\}$

$=\operatorname{INT}\{31-31 / 2-31 / 3-31 / 5+31 / 6+31 / 10+31 / 15-31 / 30+3-1\}$

$=\{31-15-10-6+5+3+2-1+3-1\}=11$

$\operatorname{Pi}(32)=\operatorname{INT}\{32 \times(1-1 / 2) \times(1-1 / 3) \times(1-1 / 5)+3-1\}$

$=\operatorname{INT}\{32-32 / 2-32 / 3-32 / 5+32 / 6+32 / 10+32 / 15-32 / 30+3-1\}$

$=\{32-16-10-6+5+3+2-1+3-1\}=11$

$\operatorname{Pi}(33)=\operatorname{INT}\{33 \times(1-1 / 2) \times(1-1 / 3) \times(1-1 / 5)+3-1\}$

$=\operatorname{INT}\{33-33 / 2-33 / 3-33 / 5+33 / 6+33 / 10+33 / 15-33 / 30+3-1\}$

$=\{33-16-11-6+5+3+2-1+3-1\}=11$

$\operatorname{Pi}(34)=\operatorname{INT}\{34 \times(1-1 / 2) \times(1-1 / 3) \times(1-1 / 5)+3-1\}$

$=\operatorname{INT}\{34-34 / 2-34 / 3-34 / 5+34 / 6+34 / 10+34 / 15-34 / 30+3-1\}$

$=\{34-17-11-6+5+3+2-1+3-1\}=11$

$\operatorname{Pi}(35)=\operatorname{INT}\{35 \times(1-1 / 2) \times(1-1 / 3) \times(1-1 / 5)+3-1\}$

$=\operatorname{INT}\{35-35 / 2-35 / 3-35 / 5+35 / 6+35 / 10+35 / 15-35 / 30+3-1\}$

$=\{35-17-11-7+5+3+2-1+3-1\}=11$

$\operatorname{Pi}(36)=\operatorname{INT}\{36 \times(1-1 / 2) \times(1-1 / 3) \times(1-1 / 5)+3-1\}$

$=\operatorname{INT}\{36-36 / 2-36 / 3-36 / 5+36 / 6+36 / 10+36 / 15-36 / 30+3-1\}$

$=\{36-18-12-7+6+3+2-1+3-1\}=11$

$\operatorname{Pi}(37)=\operatorname{INT}\{37 \times(1-1 / 2) \times(1-1 / 3) \times(1-1 / 5)+3-1\}$

$=\operatorname{INT}\{37-37 / 2-37 / 3-37 / 5+37 / 6+37 / 10+37 / 15-37 / 30+3-1\}$

$=\{37-18-12-7+6+3+2-1+3-1\}=12$

$\mathrm{Pi}(38)=\operatorname{INT}\{38 \times(1-1 / 2) \times(1-1 / 3) \times(1-1 / 5)+3-1\}$

$=\operatorname{INT}\{38-38 / 2-38 / 3-38 / 5+38 / 6+38 / 10+38 / 15-38 / 30+3-1\}$ 


$$
\begin{aligned}
& =\{38-19-12-7+6+3+2-1+3-1\}=12 \\
& \operatorname{Pi}(39)=\operatorname{INT}\{39 \times(1-1 / 2) \times(1-1 / 3) \times(1-1 / 5)+3-1\} \\
& =\operatorname{INT}\{39-39 / 2-39 / 3-39 / 5+39 / 6+39 / 10+39 / 15-39 / 30+3-1\} \\
& =\{39-19-13-7+6+3+2-1+3-1\}=12 \\
& \operatorname{Pi}(40)=\operatorname{INT}\{40 \times(1-1 / 2) \times(1-1 / 3) \times(1-1 / 5)+3-1\} \\
& =\operatorname{INT}\{40-40 / 2-40 / 3-40 / 5+40 / 6+40 / 10+40 / 15-40 / 30+3-1\} \\
& =\{40-20-13-8+6+4+2-1+3-1\}=12 \\
& \operatorname{Pi}(41)=\operatorname{INT}\{41 \times(1-1 / 2) \times(1-1 / 3) \times(1-1 / 5)+3-1\} \\
& =\operatorname{INT}\{41-41 / 2-41 / 3-41 / 5+41 / 6+41 / 10+41 / 15-41 / 30+3-1\} \\
& =\{41-20-13-8+6+4+2-1+3-1\}=13 \\
& \operatorname{Pi}(42)=\operatorname{INT}\{42 \times(1-1 / 2) \times(1-1 / 3) \times(1-1 / 5)+3-1\} \\
& =\operatorname{INT}\{42-42 / 2-42 / 3-42 / 5+42 / 6+42 / 10+42 / 15-42 / 30+3-1\} \\
& =\{42-21-14-8+7+4+2-1+3-1\}=13 \\
& \operatorname{Pi}(43)=\operatorname{INT}\{43 \times(1-1 / 2) \times(1-1 / 3) \times(1-1 / 5)+3-1\} \\
& =\operatorname{INT}\{43-43 / 2-43 / 3-43 / 5+43 / 6+43 / 10+43 / 15-43 / 30+3-1\} \\
& =\{43-21-14-8+7+4+2-1+3-1\}=14 \\
& \operatorname{Pi}(44)=\operatorname{INT}\{44 \times(1-1 / 2) \times(1-1 / 3) \times(1-1 / 5)+3-1\} \\
& =\operatorname{INT}\{44-44 / 2-44 / 3-44 / 5+44 / 6+44 / 10+44 / 15-44 / 30+3-1\} \\
& =\{44-22-14-8+7+4+2-1+3-1\}=14 \\
& \operatorname{Pi}(45)=\operatorname{INT}\{45 \times(1-1 / 2) \times(1-1 / 3) \times(1-1 / 5)+3-1\} \\
& =\operatorname{INT}\{45-45 / 2-45 / 3-45 / 5+45 / 6+45 / 10+45 / 15-45 / 30+3-1\} \\
& =\{45-22-15-9+7+4+3-1+3-1\}=14
\end{aligned}
$$

$$
\begin{aligned}
& \operatorname{Pi}(46)=\operatorname{INT}\{46 \times(1-1 / 2) \times(1-1 / 3) \times(1-1 / 5)+3-1\} \\
& =\operatorname{INT}\{46-46 / 2-46 / 3-46 / 5+46 / 6+46 / 10+46 / 15-46 / 30+3-1\} \\
& =\{46-23-15-9+7+4+3-1+3-1\}=14 \\
& \operatorname{Pi}(47)=\operatorname{INT}\{47 \times(1-1 / 2) \times(1-1 / 3) \times(1-1 / 5)+3-1\} \\
& =\operatorname{INT}\{47-47 / 2-47 / 3-47 / 5+47 / 6+47 / 10+47 / 15-47 / 30+3-1\} \\
& =\{47-23-15-9+7+4+3-1+3-1\}=15 \\
& \operatorname{Pi}(48)=\operatorname{INT}\{48 \times(1-1 / 2) \times(1-1 / 3) \times(1-1 / 5)+3-1\} \\
& =\operatorname{INT}\{48-48 / 2-48 / 3-48 / 5+48 / 6+48 / 10+48 / 15-48 / 30+3-1\} \\
& =\{48-24-16-9+8+4+3-1+3-1\}=15
\end{aligned}
$$

Three: The Limit of New Prime Number Theorem Formula

Let $\mathrm{Pi}(\mathrm{N})$ be the prime-counting function that gives the number of primes less than or equal to $\mathrm{N}$, for any real number $\mathrm{N}$. The new prime number theorem can be expressed by the formula as follows:

$\operatorname{Pi}(\mathrm{N})=\operatorname{INT}\{\mathrm{N} \times(1-1 / \mathrm{P} 1) \times(1-1 / \mathrm{P} 2) \times \ldots \times(1-1 / \mathrm{Pm})+\mathrm{m}-1\}$, where the INT $\{\ldots\}$ expresses the taking integer operation of formula spread out type in $\{\ldots\}, \mathrm{P} 1, \mathrm{P} 2, \ldots, \mathrm{Pm}$ are all prime numbers less than or equal to $\sqrt{N}$. For example:

\section{Conclusion}

According to the above formula we can obtain the formula as follows:

$\operatorname{Pi}(\mathrm{N}) \approx \operatorname{Psha}(\mathrm{N}) \equiv \operatorname{Li}(\mathrm{N}) \times(1-(1+1 /(\operatorname{Ln}(\mathrm{N})-5)) / \sqrt{N})$

$\geq \operatorname{Sha}(\mathrm{N}) \equiv 2 /(1+\sqrt{ }(1-4 / \operatorname{Ln}(\mathrm{N}))) \times \mathrm{N} / \operatorname{Ln}(\mathrm{N}) \geq \mathrm{N} /(\operatorname{Ln}(\mathrm{N})-1)$,

where $\operatorname{Li}(\mathrm{N})$ is the logarithmic integral function, the $\operatorname{Ln}(\mathrm{N})$ denotes the natural logarithm of $\mathrm{N}$. 